Journal of Statistical Physics, Vol. 11, No. 3, 1974

\title{
Quasinormal Distributions and Expansion at the Mode
}

\author{
Yukio Tomozawa ${ }^{1}$
}

\author{
Received March 29, 1974
}

\begin{abstract}
The Gram-Charlier series of type A is discussed in terms of deviants which are related to moments in a way similar to the way Hermite polynomials are related to the powers. Distribution functions are also expressed in terms of the mode and moments (cumulants or deviants), which are useful expansions when the distributions are approximately normal. It is shown that such expansions as well as the Gram-Charlier series are valid asymptotically for discrete distributions defined on the semiinfinite interval $[0, \infty]$.
\end{abstract}

KEY WORDS : Gram-Charlier series of type A ; mode ; moments ; cumulants ; deviants ; quasinormal expansion.

\section{INTRODUCTION}

It is an interesting mathematical problem to express distribution functions in terms of various moments or cumulants. The Gram-Charlier (GC) series ${ }^{(1)}$ of type $\mathrm{A}$ is one of the solutions which is useful if the distribution is approximately normal (Gaussian). ${ }^{(2,3)}$ One of the characteristics of such a distribution-which we may call a quasinormal distribution -is that it has a unique maximum, the so-called mode. In this paper we will show that knowledge of the mode enables us to derive a useful expansion for the distribution function.

The GC series and the expansion around the mode are obtained for a continuous distribution in the range $[-\infty, \infty]$. In physics problems, however, we often encounter discrete distributions which are defined in the range $[0, \infty]$. For example, the cross sections $\sigma_{n}$ for producing extra $n$ particles in

Work supported in part by the U.S. Atomic Energy Commission.

${ }^{1}$ Randall Laboratory of Physics, The University of Michigan, Ann Arbor, Michigan. 
high-energy collision are defined for the multiplicity $n=0,1,2, \ldots$. A characteristic feature of the experimental data on multiplicity distributions is that they are quasinormal ${ }^{(4-8)}$ and the mode and the width become larger as the energy increases. It appears, therefore, that the expansions which we mentioned earlier are useful for these problems, at least in an asymptotic sense. We shall show that this is indeed the case and shall describe the condition for the validity of the asymptotic expansions.

In Section 2 the GC series for distributions defined in the range $[-\infty, \infty]$ is discussed by introducing deviants, which are functions of moments or cumulants. It is pointed out that the relationship between the deviants and the moments is similar to that between the Hermite polynomials and the powers. Section 3 deals with expansions at the mode, and Section 4 deals with the problem of discrete distributions in the semiinfinite range.

\section{DEVIANTS AND THE GRAM-CHARLIER SERIES}

Moments $\mu_{k}$ and cumulants $\kappa_{k}$ for a distribution function $f(x)$ normalized in the range $-\infty<x<\infty$ are defined through the characteristic function (c.f.)

$$
\begin{aligned}
\phi(t) & =\int_{-\infty}^{\infty} e^{i t x} f(x) d x \\
& =\sum_{k=0}^{\infty} \frac{\mu_{k}(i t)^{k}}{k !}=\exp \left[\sum_{k=1}^{\infty} \frac{\kappa_{k}(i t)^{k}}{k !}\right] \\
& =e^{i \mu_{1} t}\left[1+\sum_{k=2}^{\infty} \frac{\mu_{k}(\vec{x})(i t)^{k}}{k !}\right]
\end{aligned}
$$

where

$$
\mu_{k}(a)=\overline{(x-a)^{k}}=\int_{-\infty}^{\infty}(x-a)^{k} f(x) d x
$$

denotes the $k$ th moment around a point $a$, and

$$
\mu_{k} \equiv \mu_{k}(0)
$$

Cumulants are related to moments:

$$
\kappa_{1}=\mu_{1}=\bar{x}, \quad \kappa_{2}=\mu_{2}(\bar{x}), \quad \kappa_{3}=\mu_{3}(\bar{x}), \quad \kappa_{4}=\mu_{4}(\bar{x})-3\left[\mu_{2}(\bar{x})\right]^{2}, \quad \text { etc. }
$$


It is convenient for our purpose to introduce deviants ${ }^{2} \lambda_{k}(k \geqslant 3)$ by

$$
\begin{aligned}
\phi(t) & =\left\{\exp \left[i \kappa_{1} t+\frac{\kappa_{2}(i t)^{2}}{2}\right]\right\}\left[1+\sum_{k=3}^{\infty} \frac{\lambda_{k}(i t)^{k}}{k !}\right] \\
& =\left\{\exp \left[\frac{i \kappa_{1}}{\sqrt{ } \kappa_{2}} \tilde{t}+\frac{(i \tilde{t})^{2}}{2}\right]\right\}\left[1+\sum_{k=3}^{\infty} \frac{\tilde{\lambda}_{k}(i \tilde{i})^{k}}{k !}\right]
\end{aligned}
$$

where

$$
\tilde{t}=\sqrt{\kappa_{2}} t
$$

and

$$
\tilde{\lambda}_{k}=\lambda_{k} / \kappa_{2}^{k / 2}
$$

Since the c.f.

$$
\phi(t)=\exp \left[i \kappa_{1} t+\frac{1}{2} \kappa_{2}(i t)^{2}\right]
$$

corresponds to the normal distribution

$$
\begin{aligned}
f(x) & =\frac{1}{2 \pi} \int_{-\infty}^{\infty}\left\{\exp \left[i \kappa_{1} t+\frac{\kappa_{2}(i t)^{2}}{2}\right]\right\} \exp (-i t x) d t \\
& =\frac{1}{\left(2 \pi \kappa_{2}\right)^{1 / 2}} \exp -\frac{\left(x-\kappa_{1}\right)^{2}}{2 \kappa_{2}}
\end{aligned}
$$

deviants $\lambda_{k}$ or $\tilde{\lambda}_{k}$ give the measure of deviation from the normal distribution (as do the cumulants $\kappa_{k}, k \geqslant 3$ ). Deviants are related to moments and cumulants in the following way:

$$
\begin{aligned}
& \lambda_{3}=\kappa_{3}=\mu_{3}(\bar{x}) \\
& \lambda_{4}=\kappa_{4}=\mu_{4}(\bar{x})-3\left[\mu_{2}(\bar{x})\right]^{2} \\
& \lambda_{5}=\kappa_{5}=\mu_{5}(\bar{x})-10 \mu_{3}(\bar{x}) \mu_{2}(\bar{x})
\end{aligned}
$$

and

$$
\begin{aligned}
\lambda_{k}= & k ! \sum_{l=0}^{[k / 2]} \frac{(-1)^{l} \kappa_{2}^{l} \mu_{k-2 l}(\bar{x})}{2^{l} l !(k-2 l) !}, \quad k \geqslant 3 \\
= & \kappa_{k}+\frac{k !}{2 !} \sum_{\substack{k_{1} \geq 3 \\
k_{1}+k_{2}=k}} \frac{\kappa_{k_{1}} \kappa_{k_{2}}}{k_{1} ! k_{2} !} \\
& +\frac{k !}{3 !} \sum_{\substack{k_{i} \geq 3 \\
k_{1}+k_{2}+k_{3}=k}} \frac{\kappa_{k_{1}} \kappa_{k_{2}} \kappa_{k_{3}}}{k_{1} ! k_{2} ! k_{3} !}+\cdots, \quad k \geqslant 3
\end{aligned}
$$

${ }^{2}$ This name was suggested by $G$. West. 
Using Eq. (10) and similar notation $\left(\tilde{\kappa}_{k}=\kappa_{k} / \kappa_{2}^{k / 2}\right.$, etc.), we may rewrite Eqs. (14) and (15) as

$$
\begin{aligned}
\tilde{\lambda}_{k}= & k ! \sum_{i=0}^{[k / 2]} \frac{(-1)^{2} \tilde{\mu}_{k-2 l}(\bar{x})}{2 l l(k-2 l) !} \quad k \geqslant 3 \\
= & \tilde{\kappa}_{k}+\frac{k !}{2 !} \sum_{\substack{k_{i} \geq 3 \\
k_{1}+k_{2}=3}} \frac{\tilde{\kappa}_{k_{1}} \tilde{\kappa}_{k_{2}}}{k_{1} ! k_{2} !} \\
& +\frac{k !}{3 !} \sum_{\substack{k_{i} \geq 3 \\
k_{1}+k_{2}+k_{3}=k}} \frac{\tilde{\kappa}_{k_{1}} \tilde{\kappa}_{k_{2}} \tilde{\kappa}_{k_{3}}}{k_{1} ! k_{2} ! k_{3} !}+\cdots, \quad k \geqslant 3
\end{aligned}
$$

We notice that in Eqs. (14) and (16) we have the identity

$$
\mu_{1}(\bar{x})=\tilde{\mu}_{1}(\bar{x})=0
$$

by definition, and that the series in Eqs. (15) and (17) terminates with the $[k / 3]$ th sum. The inverse of Eq. (16) is given by

$$
\tilde{\mu}_{k}(x)=k ! \sum_{i=0}^{[k / 2]} \frac{\tilde{\lambda}_{k-2 l}}{2 l !(k-2 l) !}
$$

with the constraints

$$
\tilde{\lambda}_{1}=\tilde{\lambda}_{2} \equiv 0
$$

With these preparations we express the distribution function $f(x)$ in terms of deviants: Using Eqs. (1), (8), and (9) and defining

$$
z=\left(x-\kappa_{1}\right) / \sqrt{\kappa_{2}}
$$

we obtain

$$
\begin{aligned}
f(x) & =\frac{1}{2 \pi} \int_{-\infty}^{\infty} e^{-i t x} \phi(t) d t \\
& =\left(1+\sum_{k=3}^{\infty} \frac{\tilde{\lambda}_{k}(-d / d z)^{k}}{k !}\right) \frac{1}{2 \pi \sqrt{\kappa_{2}}} \int_{-\infty}^{\infty} \exp \left(-i z \tilde{t}-\frac{\tilde{t}^{2}}{2}\right) d \tilde{t} \\
& =\frac{1}{\left(2 \pi \kappa_{2}\right)^{1 / 2}}\left(1+\sum_{k=3}^{\infty} \frac{\tilde{\lambda}_{k} H_{k}(z)}{k !}\right) \exp -\frac{z^{2}}{2}
\end{aligned}
$$

where the identity of the Hermite polynomial

$$
\left(\frac{d}{d z}\right)^{k} \exp -\frac{z^{2}}{2}=(-1)^{k} H_{k}(z) \exp -\frac{z^{2}}{2}
$$

has been used. Equation (23) is the Gram-Charlier series of type A. 
We point out that the reciprocal relation between moments and deviants, Eqs. (16) and (19), resembles that between the powers and the Hermite polynomials

$$
H_{k}(z)=k ! \sum_{i=0}^{[k / 2]} \frac{(-1)^{l} z^{k-2 l}}{2 l ! !(k-2 l) !}
$$

and

$$
z^{k}=k ! \sum_{l=0}^{[k / 2]} \frac{H_{k-2 l}(z)}{2^{2 l} l !(k-2 l) !}
$$

which follows from the generating function

$$
\exp \left(t z-\frac{t^{2}}{2}\right)=\sum_{j=0}^{\infty} \frac{t^{j} H_{j}(z)}{j !}
$$

The only difference is that in the former the first few terms of moments and deviants are missing by definition [Eqs. (8) and (20)]. Using Eqs. (25), we recast the $\mathrm{GC}$ series into the form

$$
f(x)=\frac{1}{\left(2 \pi \kappa_{2}\right)^{1 / 2}}\left(\exp -\frac{z^{2}}{2}\right) \sum_{k=0}^{\infty} \frac{\tilde{\nu}_{k} z^{k}}{k !}
$$

where

$$
\begin{gathered}
\tilde{\nu}_{0}=1+\frac{\tilde{\lambda}_{4}}{4 ! !}-\frac{\tilde{\lambda}_{6}}{6 ! !}+\frac{\tilde{\lambda}_{8}}{8 ! !}-\cdots, \quad \tilde{\nu}_{1}=-\frac{\tilde{\lambda}_{3}}{2 ! !}+\frac{\tilde{\lambda}_{5}}{4 ! !}-\frac{\tilde{\lambda}_{7}}{6 ! !}+\cdots \\
\tilde{\nu}_{2}=-\frac{\tilde{\lambda}_{4}}{2 ! !}+\frac{\tilde{\lambda}_{6}}{4 ! !}-\frac{\tilde{\lambda}_{8}}{6 ! !}+\cdots, \quad \tilde{\nu}_{3}=\tilde{\lambda}_{3}-\frac{\tilde{\lambda}_{5}}{2 ! !}+\frac{\tilde{\lambda}_{7}}{4 ! !}-\cdots
\end{gathered}
$$

and

$$
\tilde{\boldsymbol{\nu}}_{k}=\sum_{l=0}^{\infty} \frac{(-1)^{l}}{(2 l) ! !} \delta_{2 l+k}, \quad k \geqslant 3
$$

Finally we note the further relations

$$
\phi(t)=\left\{\exp \left[i \frac{\kappa_{1}}{\sqrt{\kappa_{2}}} \tilde{t}+\frac{(i \tilde{t})^{2}}{2}\right]\right\} \sum_{k=0}^{\infty} \frac{\tilde{\nu}_{k} i^{k} H_{k}(\tilde{t})}{k !}
$$

and

$$
z^{k} \exp -\frac{z^{2}}{2}=i^{k} H_{k}\left(i \frac{d}{d z}\right) \exp -\frac{z^{2}}{2}=i^{-k} H_{k}\left(\frac{1}{i} \frac{d}{d z}\right) \exp -\frac{z^{2}}{2}
$$

which are reciprocal to Eqs. (8) and (24), respectively. Equation (31) can be obtained from Eqs. (8) and (26), and Eq. (32) is the Fourier transform of 
Eq. (24). From Eqs. (22), (31), and (32) it follows that

$$
\begin{aligned}
f(x) & =\sum_{k=0}^{\infty} \frac{\tilde{\nu}_{k} i^{k} H_{k}(i d / d z)}{k !} \frac{1}{\left(2 \pi \kappa_{2}\right)^{1 / 2}} \exp -\frac{z^{2}}{2} \\
& =\frac{1}{\left(2 \pi \kappa_{2}\right)^{1 / 2}} \sum_{k=0}^{\infty} \frac{\tilde{\nu}_{k} z^{k}}{k !} \exp -\frac{z^{2}}{2}
\end{aligned}
$$

which is identical to Eq. (28).

\section{MODE AND QUASINORMAL EXPANSION}

The mode $m$ is the stationary point of distribution functions and is determined as the solution of the equation

$$
\begin{aligned}
& \sum_{k=0}^{\infty} \frac{\tilde{\nu}_{k+1} z^{k}}{k !}-z \sum_{k=0}^{\infty} \frac{\tilde{\nu}_{k} z^{k}}{k !} \\
& \quad=\tilde{\nu}_{1}+\left(\tilde{\nu}_{2}-\tilde{\nu}_{0}\right) z+\left(\frac{\tilde{\nu}_{3}}{2}-\tilde{\nu}_{1}\right) z^{2}+\cdots+\left(\frac{\tilde{\nu}_{k+1}}{k !}-\frac{\tilde{\nu}_{k-1}}{(k-1) !}\right) z^{k}+\cdots \\
& \quad=0
\end{aligned}
$$

This can be solved only if a few terms in the series are important, or if Eq. (34) is summable in a compact form. The former case was discussed in Refs. 2, 3, and 9 (also see Ref. 1, Section 6.25). In this paper, instead, we consider the case where the mode is known. This simplifies the problem enormously as far as a formal manipulation is concerned, as will be seen below.

In terms of the mode, we anticipate the expansion

$$
\begin{aligned}
f(x) & =\frac{1}{(2 \pi)^{1 / 2} \beta}\left[\exp -\frac{(x-m)^{2}}{2 \gamma^{2}}\right]\left[1+\sum_{k=3}^{\infty} \frac{a_{k}}{k !}\left(\frac{x-m}{\gamma}\right)^{k}\right] \\
& =\frac{1}{(2 \pi)^{1 / 2} \beta} \exp \left[-\frac{1}{2}\left(\frac{x-m}{\gamma}\right)^{2}+\sum_{k=3}^{\infty} \frac{b_{k}}{k !}\left(\frac{x-m}{\gamma}\right)^{k}\right]
\end{aligned}
$$

These expansion formulas were discussed previously ${ }^{(3)}$ for the Poisson distribution and temperate correlation models which are characterized by the condition

$$
\tilde{\kappa}_{k}=O\left(\epsilon^{k-2}\right), \quad k \geqslant 3
$$

where

$$
\epsilon \ll 1
$$

In the latter case we can prove that ${ }^{(3)}$

$$
a_{3}=O(\epsilon), \quad a_{4,6}=O\left(\epsilon^{2}\right), \quad a_{3 l-4,3 l-2,3 l}=O\left(\epsilon^{l}\right), \quad l \geqslant 3
$$


and

$$
b_{k}=O\left(\epsilon^{k-2}\right), \quad k \geqslant 3
$$

Thus the coefficients of higher powers in $(x-m) / \gamma$ are successively smaller. The distribution clearly exhibits a quasinormal behavior. The mode and the width are also calculated ${ }^{(2,3)}$ as expansions in $\epsilon$

$$
m=\kappa_{1}-\frac{1}{2}\left(\kappa_{3} / \kappa_{2}\right)\left[1+O\left(\epsilon^{2}\right)\right]
$$

and

$$
\gamma=\sqrt{\kappa_{2}}\left[1+O\left(\epsilon^{2}\right)\right]
$$

The aim of this section is to derive expansion formulas (35) in a more general case.

Assuming that the mode $m$ is already known, the width may be computed by the formula

$$
\frac{1}{\gamma^{2}}=-\left.\frac{\partial^{2} \ln f(x)}{\partial x^{2}}\right|_{x=m}=\frac{1}{\kappa_{2}}\left[1+z_{m}{ }^{2}-\frac{\sum_{k=0}^{\infty}\left(\tilde{\nu}_{k+2} z_{m}{ }^{k} / k !\right)}{\sum_{k=0}^{\infty}\left(\tilde{\nu}_{k} z_{m}{ }^{k} / k !\right)}\right]
$$

where

$$
z_{m}=\left(m-\kappa_{1}\right) / \sqrt{\kappa_{2}}
$$

In order to compute the other parameters in the expansion formulas (35) it would be more convenient to use the expansion of the c.f.

$$
\begin{aligned}
\phi(t) & =\left\{\exp \left[i m t+\frac{(i \gamma t)^{2}}{2}\right]\right\}\left[1+\sum_{k=1}^{\infty} \frac{\xi_{k}(i t)^{k}}{k !}\right] \\
& =\left\{\exp \left[i \frac{m}{\gamma} \hat{t}+\frac{(i \hat{t})^{2}}{2}\right]\right\}\left[1+\sum_{k=1}^{\infty} \frac{\hat{\xi}_{k}(i \hat{i})^{k}}{k !}\right]
\end{aligned}
$$

where

$$
\begin{aligned}
& \xi_{k}=k ! \sum_{l=0}^{[k / 2]} \frac{(-1)^{l} \gamma^{2 l} \mu_{k-2 l}(m)}{2^{l} l !(k-2 l) !} \\
& \xi_{k}=k ! \sum_{l=0}^{[k / 2]} \frac{(-1)^{l} \hat{\mu}_{k-2 l}(m)}{2^{l} l !(k-2 l) !} \\
& \hat{t}=\gamma t \\
& \hat{\xi}_{k}=\xi_{k} / \gamma^{k}, \quad \hat{\mu}_{k}=\mu_{k} / \gamma^{k}, \quad \text { etc. }
\end{aligned}
$$

and

$$
\mu_{0}(m)=\xi_{0}=1
$$


It is obvious that we have the relation reciprocal to Eq. (47) which is similar to Eq. (26),

$$
\hat{\mu}_{k}(m)=k ! \sum_{i=0}^{[k / 2]} \frac{\hat{\xi}_{k-2 l}}{2^{i} l !(k-2 l) !}
$$

An alternative expression of the c.f. which is analogous to Eq. (31) is given by

$$
\phi(t)=\left\{\exp \left[i \frac{m}{\gamma} \hat{t}+\frac{(i \hat{t})^{2}}{2}\right]\right\} \sum_{k=0}^{\infty} \frac{\hat{\eta}_{k} i^{k} H_{k}(\hat{t})}{k !}
$$

where the identity (26) was used, and $\hat{\eta}_{k}$ is related to $\hat{\xi}_{k}$ by

$$
\begin{aligned}
& \hat{\eta}_{0}=1+\sum_{l=1}^{\infty} \frac{(-1)^{l}}{(2 l) ! !} \hat{\xi}_{2 l} \\
& \hat{\eta}_{k}=\sum_{l=0}^{\infty} \frac{(-1)^{l}}{(2 l) ! !} \hat{\xi}_{2 l+k}, \quad k \geqslant 1
\end{aligned}
$$

As was done previously, the distribution function is obtained as the Fourier transform of Eq. (52),

$$
\begin{aligned}
f(x) & =\sum_{k=0}^{\infty} \frac{\hat{\eta}_{k} i^{k} H_{k}(i d / d y)}{k !} \frac{1}{(2 \pi)^{1 / 2} \gamma} \exp -\frac{y^{2}}{2} \\
& =\frac{1}{(2 \pi)^{1 / 2} \gamma} \sum_{k=0}^{\infty} \frac{\hat{\eta}_{k} y^{k}}{k !} \exp -\frac{y^{2}}{2}
\end{aligned}
$$

where

$$
y=(x-m) / \gamma
$$

From the fact that $m$ is the mode and $\gamma$ is the width, it follows that

$$
\hat{\eta}_{1}=\hat{\eta}_{2}=0
$$

and Eq. (55) can be written in the form (35a) with the parameter

and

$$
\beta=\gamma / \hat{\eta}_{0}
$$

$$
a_{k}=\hat{\eta}_{k} / \hat{\eta}_{0}, \quad k \geqslant 3
$$

The coefficients $b_{k}$ in Eq. (35b) can be expressed in terms of $a_{k}$ and vice versa:

and

$$
b_{k}=a_{k}-\frac{k !}{2} \sum_{\substack{k_{2} \geq 3 \\ k_{1}+k_{2}=k}} \frac{a_{k_{1}} a_{k_{2}}}{k_{1} ! k_{2} !}+\frac{k !}{3} \sum_{\substack{k_{i} \geq 3 \\ k_{1}+k_{2}+k_{3}=k}} \frac{a_{k_{1}} a_{k_{2}} a_{k_{3}}}{k_{1} ! k_{2} ! k_{3} !}-\cdots
$$

$$
a_{k}=b_{k}+\frac{k !}{2 !} \sum_{\substack{k_{i} \geq 3 \\ k_{1}+k_{2}=k}} \frac{b_{k_{1}} b_{k_{2}}}{k_{1} ! k_{2} !}+\frac{k !}{3 !} \sum_{\substack{k_{i} \geq 3 \\ k_{1}+k_{2}+k_{3}=k}} \frac{b_{k_{1}} b_{k_{2}} b_{k_{3}}}{k_{1} ! k_{2} ! k_{3} !}+\cdots
$$


The series in Eqs. (60) and (61) terminates with the [k/3]th sum as in Eq. (17). This completes the formal derivation of the quasinormal expansion, Eqs. (35). Needless to say, such expansions are most effective if $a_{k}$ or $b_{k}$ decreases very quickly as $k \rightarrow \infty$.

\section{DISCRETE DISTRIBUTION IN THE SEMIINFINITE RANGE ${ }^{3}$}

For a discrete distribution $P_{n}$ which is normalized by

$$
\sum_{n=0}^{\infty} P_{n}=1
$$

we proceed in a way similar to the preceding sections. The c.f. is defined by

$$
\phi(t)=\sum_{n=0}^{\infty} e^{i n t} P_{n}
$$

and most of the formulas concerning the moments, cumulants, deviants, and the like, are valid also in this case except that the integral in $x$ is replaced by the sum over $n$. For example, the moments are given by

$$
\mu_{k}(a)=\overline{(n-a)^{k}}=\sum_{n=0}^{\infty}(n-a)^{k} P_{n}
$$

Using Eqs. (8)-(10) and (31), we invert Eq. (63) to obtain

$$
\begin{aligned}
P_{n} & =\frac{1}{2 \pi} \int_{-\pi}^{\pi} e^{-i n t} \phi(t) d t \\
& =\left[1+\sum_{k=3}^{\infty} \frac{\tilde{\lambda}_{k}(-d / d z)^{k}}{k !}\right] \frac{1}{2 \pi \sqrt{\kappa_{2}}} \int_{-\pi^{\sqrt{\kappa_{2}}}}^{\pi^{\sqrt{ }} \overline{k_{2}}} \exp \left(-i z \tilde{t}-\frac{\tilde{t}^{2}}{2}\right) d \tilde{t} \\
& =\sum_{k=0}^{\infty} \frac{\tilde{\nu}_{k} i^{k} H_{k}(i d / d z)}{k !} \frac{1}{2 \pi \sqrt{\kappa_{2}}} \int_{-\pi^{\sqrt{k_{2}}}}^{\pi^{\sqrt{\kappa_{2}}}} \exp \left(-i z \tilde{t}-\frac{\tilde{t}^{2}}{2}\right) d \tilde{t}
\end{aligned}
$$

where

$$
z=\left(n-\kappa_{1}\right) / \sqrt{\kappa_{2}}
$$

The integral in the above equations is

$$
\begin{aligned}
\chi(n) & =\frac{1}{2 \pi \sqrt{\kappa_{2}}}\left(\exp -\frac{z^{2}}{2}\right) \int_{-\pi \sqrt{\kappa_{2}}}^{\pi^{\sqrt{\kappa_{2}}}} \exp \left[-\frac{1}{2}(\tilde{t}+i z)^{2}\right] d \tilde{t} \\
& =\frac{1}{\left(2 \pi \kappa_{2}\right)^{1 / 2}}\left(\exp -\frac{z^{2}}{2}\right) \operatorname{Re}\left\{\operatorname{Erf}\left[\pi\left(\frac{\kappa_{2}}{2}\right)^{1 / 2}\left(1+\frac{i\left(n-\kappa_{1}\right)}{\pi \kappa_{2}}\right)\right]\right\}
\end{aligned}
$$

${ }^{3}$ The basic argument in this section is the same as in Ref. 3 . We present it for completeness to include the case of the general expansion formula discussed in the previous sections. 
where the error function and its asymptotic form are given by

$$
\begin{aligned}
& \operatorname{Erf}(u)=\frac{2}{\sqrt{\pi}} \int_{0}^{u} \exp -t^{2} d t \\
& \underset{u \rightarrow \infty}{\longrightarrow} 1-\frac{1}{\sqrt{\pi}} \frac{\exp -u^{2}}{u}\left[1+O\left(\frac{1}{u^{2}}\right)\right]
\end{aligned}
$$

In the limit $\kappa_{2} \rightarrow \infty$, therefore, we get the asymptotic expression for $\chi(n)$

$$
\begin{aligned}
\chi(n) \underset{\kappa_{2} \rightarrow \infty}{\longrightarrow} & \frac{1}{\left(2 \pi \kappa_{2}\right)^{1 / 2}}\left(\exp -\frac{z^{2}}{2}\right) \\
& \times\left\{1-O\left(\kappa^{-1 / 2} \exp -\left\{\frac{\kappa_{2}}{2}\left[\pi^{2}-\left(\frac{n-\kappa_{1}}{\kappa_{2}}\right)^{2}\right]\right\}\right)\right]
\end{aligned}
$$

The second term in the outside curly braces of Eq. (72) is negligible in the limit $\kappa_{2} \rightarrow \infty$, provided

$$
\left|\left(n-\kappa_{1}\right) / \kappa_{2}\right|<\pi
$$

Hence, Eqs. (65) and (66) coincide with Eqs. (23) and (33) asymptotically.

It is easy to see also that the asymptotic expansion

$$
\begin{aligned}
P_{n}= & \frac{1}{(2 \pi)^{1 / 2} \beta}\left[\exp -\frac{(n-m)^{2}}{2 \gamma^{2}}\right]\left[1+\sum_{k=3}^{\infty} \frac{a_{k}}{k !}\left(\frac{n-m}{\gamma}\right)^{k}\right] \\
& \times\left\{1+o\left(\gamma^{-1} \exp \left\{-\frac{\gamma^{2}}{2}\left[\pi^{2}-\left(\frac{n-m}{\gamma^{2}}\right)^{2}\right]\right\}\right)\right] \\
= & \frac{1}{(2 \pi)^{1 / 2} \beta} \exp \left[-\frac{(n-m)^{2}}{2 \gamma^{2}}+\sum_{k=3}^{\infty} \frac{b_{k}}{k !}\left(\frac{n-m}{\gamma}\right)^{k}\right] \\
& \times\left\{1+O\left(\gamma^{-1} \exp \left\{-\frac{\gamma^{2}}{2}\left[\pi^{2}-\left(\frac{n-m}{\gamma^{2}}\right)^{2}\right]\right\}\right)\right\}
\end{aligned}
$$

is valid in the limit $\gamma \rightarrow \infty$, provided the condition

$$
\left|(n-m) / \gamma^{2}\right|<\pi
$$

is satisfied. The formulas expressing the parameters of the asymptotic expansions (74) and (75) in terms of the mode and the moments are identical to those in Section 3.

\section{ACKNOWLEDGMENT}

The author is indebted to Prof. S. D. Drell for his warm hospitality extended to him at SLAC. Thanks are also due to W. Colglazier for reading the manuscript. 


\section{REFERENCES}

1. M. G. Kendall and A. Stuart, The Advanced Theory of Statistics, Vol, 1, Charles Griffin and Co., London (1963).

2. J. B. S. Haldane, Biometrika 32:294 (1942).

3. Y. Tomozawa, Phys. Rev. D 8:2138 (1973); L. Heiko and J. Hvegholm, "Charged Prong Distributions in Proton-Proton Collisions in the $50-300 \mathrm{GeV} / \mathrm{c}$ Momentum Range Using the Gram-Charlier Expansion," preprint (1973).

4. G. D. Kaiser, Nucl. Phys, B44:171 (1972); "A Comparative Study of Simple Statistical Models for Charged Particle Multiplicities," DNPL/P125 (1972); " The Approximately Normal Model for Charged Pair Production: A Reconsideration," DNPL/ P148 (1973).

5. P. Olesen, Phys. Letters 41B:602 (1972).

6. P. Slattery, Phys. Rev. D 7:2073 (1973).

7. G. W. Parry and P. Rotelli, "Application of the Truncated Gaussian to the Inelastic pp Charged Multiplicity Distribution," Trieste, IC/73/3.

8. Y. Tomozawa, Nucl. Phys. B62: 539 (1973); Phys. Rev. D 8:2319 (1973).

9. E. A. Cornish and R. A. Fisher, Rev. Inst. Int. Statist. 5:307 (1937); Technometrics 2:209 (1960). 